\title{
Does the Use of IV Contrast Enhanced CT for Attenuation Correction Affect Clinical Interpretation of Head and Neck PET/CT?
}

\author{
Lance T. Hall, Aaron F. Struck* , Christopher G. Guglielmo, Christine J. Jaskowiak, Michael A. Wilson \\ and Scott B. Perlman
}

\begin{abstract}
Department of Radiology, Section of Nuclear Medicine, University of Wisconsin, Box 3252 Clinical Science Center-E3, 600 Highland Ave Madison, WI 53792, USA
\end{abstract}

\begin{abstract}
Purpose of Report: Evaluate the effect of IV contrast use with FDG PET/CT on clinical interpretation of PET images for head and neck cancer.

Procedures: 20 consecutive patients referred for PET/CT and contrast enhanced CT of the head and neck had two sets of PET images obtained. One set used standard low dose CT for attenuation correction, and the other used IV contrast enhanced diagnostic quality CT for attenuation correction. Two blinded nuclear medicine physicians interpreted the results.

Results: No statistically significant difference in clinical interpretation of the PET images was found. It was noted that PET scans attenuation corrected with IV contrast CTs had greater incidence of abnormal appearing lesions $(\mathrm{P}<0.01)$.

Conclusion: It is reasonable to use contrast enhanced CT for attenuation correction in PET/CT of head and neck cancer. This would allow for a reduction in radiation dose delivered to patient.
\end{abstract}

Keywords: PET/CT, head and neck cancer, contrast, IV contrast, low dose CT, attenuation correction.

\section{INTRODUCTION}

Positron emission tomography with non-contrast computed tomography (PET/CT) has become a critical tool in evaluating cancer patients. It provides unique functional data not found with other imaging modalities. In many instances PET/CT has greater sensitivity and specificity in detecting primary tumors, nodal spread, and distant metastasis than MRI or CT [1]. Despite its often superior accuracy, PET/CT lacks the anatomic resolution obtained with other cross sectional imaging modalities. The low-dose non-contrast CT (LDCT) typically obtained with the PET scan is obtained primarily for attenuation correction and lesion localization. It does not provide sufficient anatomic detail necessary for diagnosis and follow-up for several cancer types including head and neck cancer $[2,3]$. The fine anatomic detail needed to investigate the anatomically complex area of the head and neck requires cross sectional imaging with higher resolution and IV contrast to define vascular structures and lymph nodes. The most common imaging technique used to obtain this level of resolution is contrast enhanced computed tomography (CECT).

Despite the potential advantages of obtaining PET and CECT together (decreased overall patient radiation dose, improved scanner utilization, and enhanced image correlation) the method is not without controversy. An area of consternation remains. It is currently unclear whether the

\footnotetext{
*Address correspondence to this author at the Department of Radiology, Section of Nuclear Medicine, University of Wisconsin, Box 3252 Clinical Science Center-E3, 600 Highland Ave Madison, WI 53792, USA; Tel: (608) 263-5585; Fax: (608) 265-7390; E-mail: astruck@uwhealth.org
}

adverse effects from CT attenuation artifacts potentially seen with CECT will significantly affect clinical interpretation of PET/CT studies in the head and neck. It is known that standard CT to PET attenuation correction algorithms are affected by high atomic weight contrast [4]. CT scans (CECT and LDCT) measure attenuation values of tissues and substances in the body with a polychromatic x-ray beam with a mean photon energy in the range of $70 \mathrm{keV}$. These attenuation values must be scaled to the PET energy of 511 $\mathrm{keV}$ for the PET/CT $[5,6]$. CT range photons interact with high atomic weight materials (e.g. IV contrast) differently than PET range photons. The relationship between the attenuation coefficients that holds true in low atomic weight materials (water, muscle, connective tissue) is distorted in high atomic weight materials. Photoelectric absorption predominates in high atomic weight materials, but low photon energies are more likely to be attenuated via this interaction [7]. This results in the CT photons having high attenuation coefficients (CT number or Hounsfield Units) that inappropriately predict high attenuation of PET range photons. In physical reality PET range photons do not have this level of attenuation. This mismatch results in overcorrection in the PET images, though new algorithms are being investigated to correct this problem [8-10]. Changing vascular contrast density throughout the PET and CT acquisitions adds an additional element of variability that is not easily corrected computationally, though specific contrast delays allows for consistency. It remains unclear if IV contrast has enough of an effect to cause clinically significant difference in interpretation of PET/CT images.

During periods of pre-treatment planning and restaging, PET/CT and CECT are often obtained separately in the same 
day or week. Theoretically the CECT can provide more clinically relevant anatomic data than the LDCT can provide. Is it possible then to forgo the LDCT? By omitting an unnecessary CT scan a patient's radiation dose is decreased and the stand-alone CT scanner is freed for other clinical use. A more direct comparison between the two imaging modalities (hardware vs software image fusion) is also achieved by obtaining the CECT and PET in the same session, potentially improving diagnostic accuracy by reducing misregistration artifacts. This prompted us to further investigation to see if CECT coincident with the PET scan was possible without loss in diagnostic accuracy.

No known studies to date have specifically evaluated how using CECT scans for attenuation correction of PET images affects the clinical interpretation of the PET images in patients with head and neck cancers. These cancers are of particular concern because of the region's highly vascular and detailed anatomy, making them more likely to have differences in PET interpretation. The primary purpose of this study is to determine if there is a statistically significant difference in the final interpretation of PET studies using attenuation correction with traditional LDCT verses "diagnostic" CECT in patients with head and neck cancers. If there is no significant difference between the two techniques, then the radiation exposure the patient receives can be reduced by eliminating the LDCT.

\section{MATERIALS AND METHODS}

\section{Ethics}

An application for human research was made to the University of Wisconsin Institutional Review Board (IRB) for this prospective quality control project. An exemption was granted because the standard clinical imaging protocol was not altered and no patient identifiable information was obtained or reviewed.

\section{Data Collection}

Twenty consecutive clinical patients with a head and neck cancer undergoing routine PET/CT imaging and diagnostic contrast enhanced CT scan (CECT) were included. Patients were instructed to fast for at least 6 hours prior to the PET/CT scan. $0.14 \mathrm{mCi} / \mathrm{kg}$ of $\mathrm{F}-18$ fluorodeoxyglucose (FDG) was injected intravenously followed by an average uptake period of 45 minutes. Patients were asked to void prior to imaging. Next, a low dose noncontrast CT (LDCT) scan (GE Discovery LS PET/CT scanner, $110 \mathrm{~mA}, 140 \mathrm{kvP}$ ) was performed from the skullbase to the midthighs as routine protocol for attenuation correction of PET images and for image correlation. Immediately following the LDCT, PET emission images were acquired from the midthighs to the skullbase. (69 images, 18 detector rings with 12,096 crystals, Crystal size $4 \times 8 \times 30 \mathrm{~mm}$, Transaxial FOV $60 \mathrm{~cm}$, Axial FOV $15.2 \mathrm{~cm}$ ) Next, $100 \mathrm{ml}$ of intravenous CT contrast (Iohexol $300 \mathrm{mgI} / \mathrm{mL}$ ) were given at a rate of $3 \mathrm{ml} / \mathrm{sec}$. Prior to obtaining contrast enhanced CT (CECT) transmission images, a 40 second delay was implemented to decrease contrast density in the major vessels and allow a more uniform distribution of contrast while still permitting adequate visualization of vascular structures. Diagnostic CECT images were obtained from the aortic arch to the skullbase using CT with field of view of $50 \mathrm{~cm}$ with $300 \mathrm{~mA}$ $140 \mathrm{kvP}$ with $1.0 \mathrm{sec}$ rotation, 4 detector rows, $5.0 \mathrm{~mm}$ thickness Pitch 0.75 : 1 at a speed $7.5 \mathrm{~mm} /$ rot. Immediately after the CECT images were acquired, a second limited PET emission scan was obtained from the aortic arch to skullbase. A second PET emission scan was required because one set of PET images could not be processed with more than one CT scan for attenuation correction due to software processing constraints of the manufacturer. Additionally, this technique would also minimize patient motion artifacts between the two PET scans. The total time between the first and second PET emission scan was 5-10 minutes.

The LDCT images were used for attenuation correction of the first PET emission scan from the skullbase to the midthighs; however, only the PET images from the aortic arch to the skullbase were displayed for interpretation in this study. The CECT images were used for attenuation correction of the second PET emission scan from the aortic arch to the skullbase. The attenuation corrected PET only images (without the accompanying CT images or fused PET/CT images) for both the LDCT and CECT methods were randomly labeled so that the readers would be blinded to the attenuation correction method used.

Two experienced readers, each with over 20 years experience, interpreted the attenuation corrected PET images independently. Readers were blinded to method used, patient's clinical history, and CT findings. Neither LDCT nor CECT images were provided for review to the interpreters. Both LDCT and CECT corrected images were randomly included in both reading sessions to ensure readers were blinded. The first set of randomized PET images was interpreted for each patient. Following a minimum 2 week delay, the second set of randomized PET images for each patient was interpreted without knowledge of the results of the first interpretation.

Foci of increased metabolic activity were graded visually as L0 for normal uptake (i.e. normal thyroid uptake, physiological muscle uptake, salivary glands, etc), L1 for indeterminate uptake (equivocal foci that could not be categorized as definitely normal or definitely abnormal such as sites of inflammation, possible recent surgical site, brown fat, etc), and L2 for definitely abnormal (obvious tumor site or very suspicious for metastasis). An overall impression was also given for each image set as 0 for normal scan without evidence for a neoplastic process, 1 for indeterminate/equivocal scan, and 2 for definite active neoplastic process. These results were recorded at the time of interpretation and the foci of interest were also labeled on an anatomic map to allow for direct comparison of scans.

\section{Data Analysis}

The number of lesions [normal (L0), equivocal (L1), and abnormal (L2)] and the overall image interpretation [normal 0 , equivocal 1, abnormal 2] were collected. For each subject (1-20) 4 data sets were obtained, two from each image reader (LDCT and CECT). The power to detect a two-sided 20\% difference in proportion of "abnormal" images between CECT and LDCT with alpha set at 0.05 was calculated to be 0.7 using JMP 8.0.2 software (copyright SAS Institute Inc., located at SAS Campus Drive, Cary, NC, USA). 
A table comparing the 4 overall impressions for each subject was created for visual inspection. Statistical analysis was carried out using a $2 \times 3$ Fisher-Freeman-Halton exact test [11] comparing LDCT and CECT for significant differences in the "overall impression" $(0,1,2)$. This test was carried out with both image readers separately and combined. The alpha level was set at 0.05 . A paired student T-test was done to compare LDCT and CECT for number of L0, L1, and L2 lesions. Again the alpha level was set at 0.05. Three tables reflecting these findings were compiled and are cited in the results section.

\section{RESULTS}

The data was collected from 20 consecutive patients (10 male, 10 female). They had an age range of 41 to 78 . The overall impressions between the images processed with LDCT and CECT did not vary greatly. Of the forty comparisons between LDCT and CECT only 4/40 had any difference, and of these only one had a downgrade of a lesion from abnormal (2) to equivocal (1). Table 1 shows a direct comparison of the overall impressions for each of the 20 subjects. Reader 1's overall impressions for the LDCT based PET were 8 normal, 1 equivocal, and 11 abnormal. Overall impressions for CECT yielded 7 normal, 2 equivocal, and 11 abnormal. The overall impressions for Reader 2 were 7 normal, 3 equivocal, and 10 abnormal for

Table 1. Comparison of Overall Interpretation of PET Images with Attenuation Correction Based on Either CECT or LDCT for Both Readers

\begin{tabular}{|c|c|c|c|c|}
\hline \multirow{2}{*}{ Patient \# } & \multicolumn{2}{|c|}{ Reader 1 } & \multicolumn{2}{c|}{ Reader 2 } \\
\cline { 2 - 5 } & CECT & LDCT & CECT & LDCT \\
\hline \hline 1 & 2 & 2 & 2 & 2 \\
\hline 2 & 0 & 0 & 0 & 0 \\
\hline 3 & 2 & 2 & 2 & 2 \\
\hline 4 & 2 & 2 & 2 & 2 \\
\hline 5 & 2 & 2 & 2 & 2 \\
\hline 6 & 0 & 0 & 1 & 1 \\
\hline 7 & 0 & 0 & 2 & 1 \\
\hline 8 & 2 & 2 & 1 & 1 \\
\hline 9 & 2 & 2 & 1 & 0 \\
\hline 10 & 0 & 0 & 0 & 0 \\
\hline 11 & 2 & 2 & 2 & 2 \\
\hline 12 & 0 & 0 & 0 & 0 \\
\hline 13 & 2 & 2 & 2 & 2 \\
\hline 14 & 0 & 0 & 0 & 0 \\
\hline 15 & 2 & 2 & 2 & 2 \\
\hline 16 & 2 & 2 & 2 & 2 \\
\hline 17 & 1 & 1 & 2 & 2 \\
\hline 18 & 2 & 2 & 2 & 2 \\
\hline 19 & 0 & 0 & 0 & 0 \\
\hline 20 & 1 & 0 & 1 & 0 \\
\hline & & & & 2 \\
\hline
\end{tabular}

the LDCT based PET, and 5 normal, 4 equivocal, and 11 abnormal by CECT based PET. It should be noted that no final interpretation changed from a "normal" to an "abnormal" or vice versa.

Fig. (1) is a representative sample of the images obtained. It compares images taken from a patient with squamous cell cancer of the supraglottis. The top images are all derived from the CECT protocol and the bottom are from the LDCT. The far right images demonstrate the similarity of the resultant attenuation corrected PET images. The CECT and the CECT with the PET overlay show improvement in tissue delineation compared with the below LDCT. The CECT/PET overlay seen in Fig. (1) is an example of PET/CECT hardware image co-registration, not the software fusion that would typically be utilized if PET data was overlayed on a contrast enhanced "diagnostic" CT taken at a different session. It should be noted that during the study the readers were blinded to all CT findings (LDCT and CECT). Statistical analysis was used to confirm the findings noted during the initial observation.

A Fisher-Freeman-Halton exact test was utilized to see if any significant difference exists in the overall impressions between the LDCT and CECT derived PET images. Analysis yielded no significant differences. The resultant $\mathrm{p}$-values are 0.90, 0.76, 0.62 for Reader 1, Reader 2, and Reader 1+ Reader 2 (respective). Further analysis was performed to evaluate inter-reader variability: no significant difference was found between Reader 1 and 2 in evaluating LDCT or CECT based PET images with p-values of 0.89 and 0.74 respectively. Table 2 lists the proportion of images placed into each category with 0 indicating normal scan, 1 being equivocal, and 2 indicating a clearly abnormal scan. The proportions remained relatively consistent between readers and between LDCT and CECT groupings.

A trend was noted that more abnormal and equivocal lesions tended to occur with CECT based PET. Reader 1 found a total of 31 lesions ( 6 equivocal and 25 abnormal) on LDCT attenuation corrected PET images, and 37 lesions (6 equivocal and 31 abnormal) on CECT attenuation corrected PET images. Reader 2 found 32 lesions (13 equivocal and 19 abnormal) by the LDCT technique, and 40 lesions (18 equivocal and 22 abnormal) by CECT technique. A paired ttest was used to compare the number of "abnormal" lesions (those graded as a "L2") identified using the two different techniques. There was a statistically significant increase in the number of abnormal lesions seen on CECT verses LDCT technique $\mathrm{p}<0.01$, while no significant difference was found in L0 or L1 lesions. Fig. (2) is a representation of these findings. The images are all taken from the same $\mathrm{H} \& \mathrm{~N}$ cancer patient. The PET images based on the CECT (a,b,c,d) show an increased number of lesions (marked with arrows) compared to the LDCT images $(e, f, g, h)$. Reader 1 found 6 abnormal lesions with CECT and 5 abnormal lesions with LDCT. Reader 2 found 3 abnormal and 2 equivocal lesions with CECT and 2 abnormal and 3 equivocal lesions with LDCT. The clinical interpretation was the same (abnormal) for both CECT and LDCT derived images.

\section{DISCUSSION}

Head and neck cancer patients were chosen for this study because of the complex anatomy and associated large 

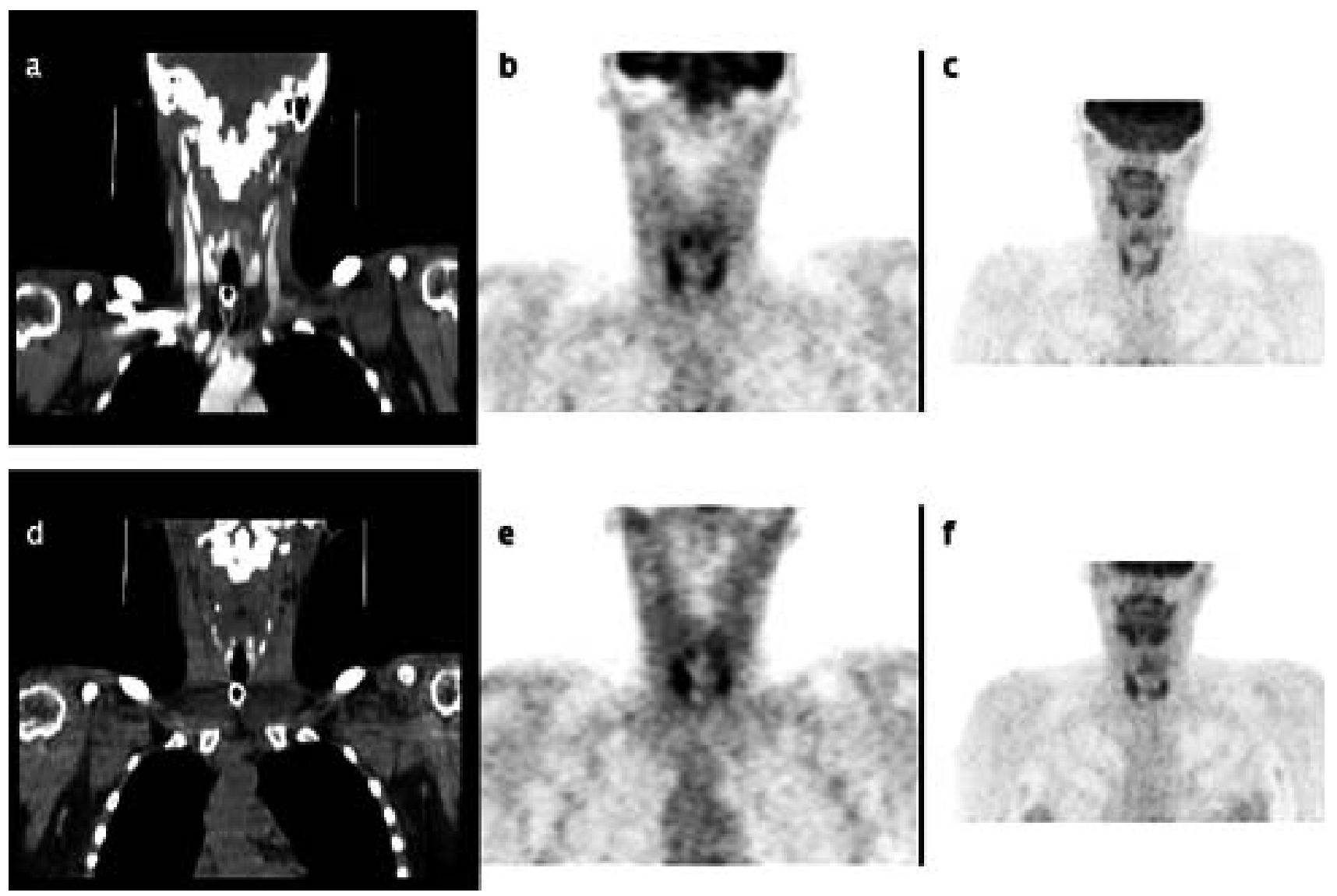

Fig. (1). This is a patient with squamous cell cancer of the supraglottis the upper half of images is obtained with CECT and the lower half is the LDCT series, (a) shows a coronal reconstruction of contrast enhanced CT, (b) CECT corrected PET, (c) is CECT corrected maximum intensity projection (MIP) PET, (d) is coronal reconstruction of LDCT, (e) is LDCT corrected PET, (f) is LDCT corrected MIP PET.

Table 2. Precentage of PET Images Read as Normal, Abnormal, or Equivocal Based on Reader and Source of Attenuation Correction (CECT vs LDCT)

\begin{tabular}{|c|c|c|c|c|c|c|}
\hline \multirow{2}{*}{ Image Interpretation } & \multicolumn{2}{|c|}{ Reader 1} & \multicolumn{2}{|c|}{ Reader 2} & \multicolumn{2}{|c|}{ Readers Combined } \\
\hline & CECT & LDCT & CECT & LDCT & CECT & LDCT* \\
\hline 0 & $35 \%$ & $40 \%$ & $25 \%$ & $35 \%$ & $30 \%$ & $38 \%$ \\
\hline 1 & $10 \%$ & $5 \%$ & $20 \%$ & $15 \%$ & $15 \%$ & $10 \%$ \\
\hline 2 & $55 \%$ & $55 \%$ & $55 \%$ & $50 \%$ & $55 \%$ & $53 \%$ \\
\hline P-value & & 0.901 & & 0.763 & & 0.629 \\
\hline
\end{tabular}

vascular structures with their proportionately large blood pool, making this region ideally sensitive to IV contrast induced artifacts. If studies can be accurately interpreted with the CECT technique in this region, then theoretically this technique can be extrapolated to other regions with less detailed anatomy. This study demonstrated that even in the anatomically complex and highly vascular region, IV contrast did not affect the overall interpretation of PET images. An increased tendency to report more lesions (equivocal and abnormal) with the CECT technique (IV contrast) was noted, but there appears to be no clinical importance of this finding regarding overall study interpretation.
Table 3. Comparison of Number of Lesions Found on PET Imaging Based on Origin of Attenuation Correction

\begin{tabular}{|c|c|c|c|}
\hline & CECT & LCDT & p-Value \\
\hline \hline L0 & 4 & 10 & 0.11 \\
\hline L1 & 24 & 19 & 0.28 \\
\hline L2 & 53 & 44 & $\mathbf{0 . 0 0 8}$ \\
\hline
\end{tabular}

The discrepancies in lesion number occurred only in patients with multiple lesions. One possible reason for this finding is contrast accumulation in both neoplastic and nonneoplastic tissues that may have contributed to an 


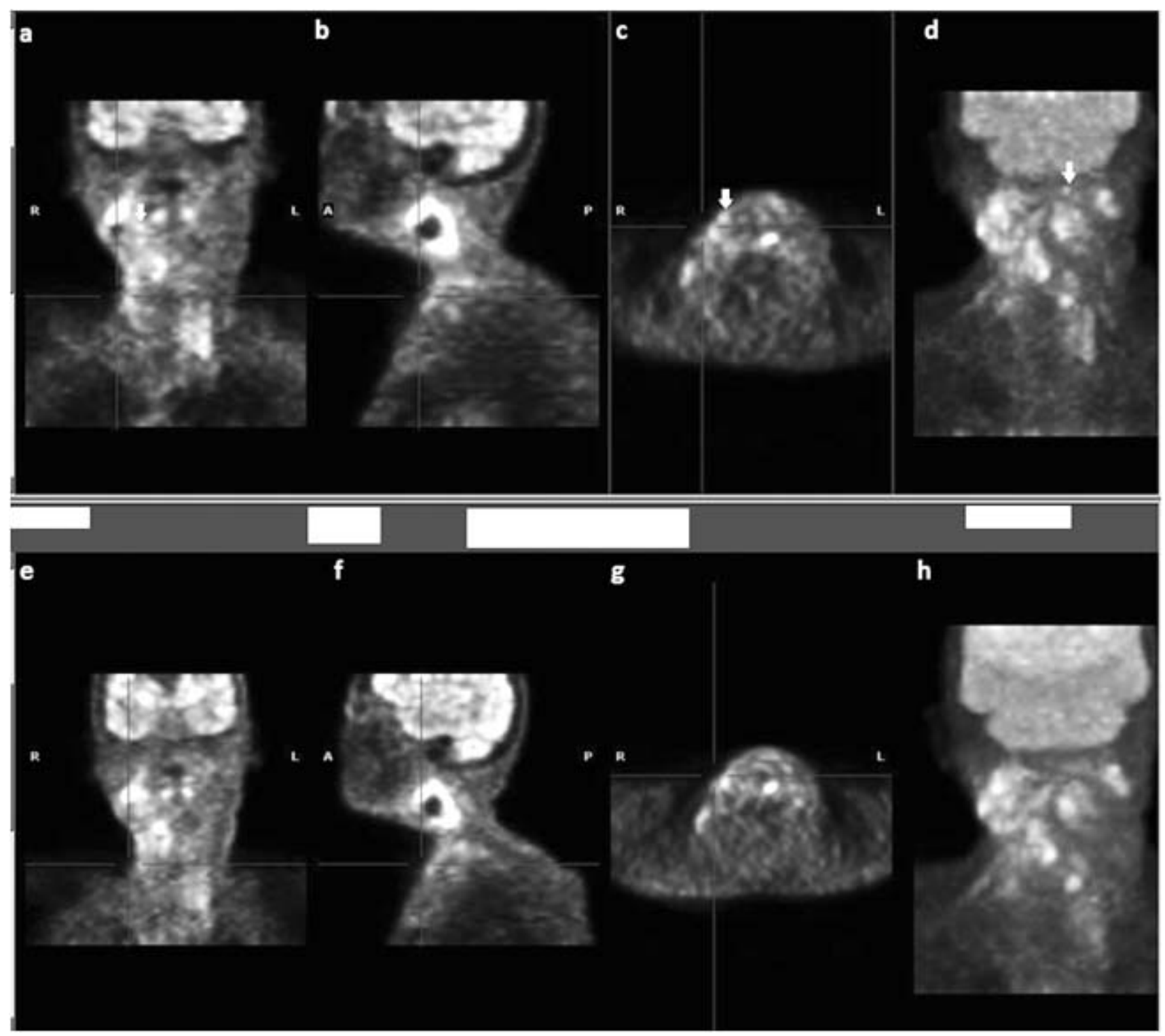

Fig. (2). This is a patient with squamous cell H\&N cancer. Images a (coronal), b (sagittal), c (axial), d (MIP) are PET images corrected with CECT; e (coronal), f (sagittal), $\mathbf{g}$ (axial), h (MIP) are PET images corrected with LDCT, the arrows indicate lesions that appear different on CECT PET. Combined the readers noted 2 L1 and 9 L2 lesions with the CECT PET, and 3 L1 and 7 L2 lesions with LDCT PET.

overestimation of apparent reconstructed activity concentration. Another potential contributor to this finding is the fact that a 5-10 minute delay occurred between the first PET emission scan (attenuation correction with the LDCT) and the second PET emission scan (attenuation correction with the CECT). During this delay, although relatively brief, continued FDG uptake may have occurred in neoplastic lesions, which may have become noticeable only on the "delayed" PET scan. Importantly, there was no statistically significant difference in the final interpretation of the PET studies using either the LDCT or CECT scan for attenuation correction.

All interpretations that were altered by the CECT technique were changed from a " 0 " (normal) to a " 1 " (equivocal) or from a " 1 " (equivocal) to a " 2 " (abnormal). It is unknown which technique, if either, is more accurate.
Perhaps equivocal/abnormal lesions are underestimated with the LDCT technique, overestimated by the CECT, or a combination of both. The major limitation in this study is the lack of a definite standard (i.e. tissue from each discrepant lesion). It cannot be definitively known which method is more accurate unless repeat studies are correlated with surgical and pathologic findings. It is known that PET/CT is a reliable and accurate test for head and neck cancer. Recent studies report the accuracy of PET/CT in H\&N cancer to be in the range of $94-96 \%[12,13]$. The important finding in this study is that the CT attenuation correction obtain with the CECT does not affect the overall interpretation of the PET scan. Similar studies have already demonstrated that the accuracy (93-95\%), sensitivity, and specificity of PET/CT based on CECT is on par with standard PET/CT $[14,15]$. 
Several recent studies have looked into the question of using contrast with PET/CT in places other than the H\&N. Studies have investigated human subjects as well as phantoms with the use of IV and oral contrast. They have generally found satisfactory results [16-22]. Mawlawi et al concluded that the use of CECT for attenuation correction of PET images did not affect the overall clinical interpretation in nine patients with intrathoracic malignancies [9]. Rodriquez-Vigil et al prospectively compared the use of CECT and LDCT for attenuation correction of 47 lymphoma patients and did not find any significant artifacts or diagnostic problems related to the use of oral or intravenous contrast material [23]. Juergens et al evaluated CECT attenuation corrected PET images in 205 patients with different malignant tumors. They concluded that qualitative assessment of PET data sets did not reveal any artifacts that limited clinical interpretation of the PET scan [24].

A few studies have looked specifically at the head and neck region. Rodrigues et al looked at 44 patients with head and neck cancer. They compared whole body PET/CT to a dedicated head and neck PET/CT sequence. The dedicated $\mathrm{H} \& \mathrm{~N}$ PET/CT used IV contrast, thinner slice CT, and smaller PET pixel size. They found that the dedicated H\&N PET/CT had similar primary tumor localization, but improved nodal metastasis detection (p-values 0.896, 0.790 respectively) [15]. Yamamoto et al found similar results without using contrast in the dedicated H\&N PET/CT portion [25]. Yoshida et al questioned whether contrast was needed at all in the evaluation of head and neck cancer patients. They compared 40 patients with whole body PET/CT with and without contrast as well as dedicated head and neck contrast enhanced CT and dedicated head and neck MRI. They found that PET/CT was superior to CT or MRI, but that no significant difference between PET/CT with or without contrast was noted in detection of primary or nodal disease [14]. These studies demonstrate that IV contrast does not adversely affect the sensitivity and specificity of PET/CT, but do not directly address if isolated PET interpretation is affected by the different attenuation correction obtained with LDCT vs CECT.

With the growing concern for excessive radiation exposure from unnecessary CT scans it is important for physicians to examine simplifying measures. This study demonstrates that using IV contrast does not affect the overall interpretation of PET images in the head and neck. Our findings are concordant with studies done in other anatomic regions including the chest and abdomen. This consensus of data along with the recent studies indicating PET/CT with IV contrast is as sensitive and specific as standard PET/CT making clinical practice with this technique a reasonable option.

\section{CONCLUSION}

No statistically significant difference in the final interpretation between PET scans using attenuation correction with CECT and LDCT was found, although a tendency to identify more equivocal and abnormal lesions on PET scans utilizing CECT for attenuation correction was noted. The clinical significance of these discrepant lesions is unknown, but additional anatomic information provided from a contrast-enhanced CT scan performed with the PET scan could presumably improve diagnostic accuracy on a lesion basis. Using CECT for attenuation correction has the potential benefits of reduction in patient radiation exposure, reduction of time in department, and reduction of time on the scanner, and CECT attenuation correction does not appear to affect the overall interpretation of the PET image. In summary, this study indicates utilizing CECT for attenuation correction of PET images in patients with head and neck cancer is a reasonable alternative to repeat CT scan with LDCT.

\section{ACKNOWLEDGEMENT}

None.

\section{CONFLICT OF INTEREST STATEMENT}

The authors declare that they have no conflict of interest.

\section{REFERENCES}

[1] Wahl RL. Principles and practive of PET and PET/CT. 2nd ed. Philadelphia: Lippincott Williams \& Wilkins 2009.

[2] Dammann F, Horger M, Mueller-Berg M, et al. Rational diagnosis of squamous cell carcinoma of the head and neck region: comparative evaluation of CT, MRI, and 18FDG PET. AJR Am J Roentgenol 2005; 184(4): 1326-31.

[3] Schwartz DL, Ford E, Rajendran J, et al. FDG-PET/CT imaging for preradiotherapy staging of head-and-neck squamous cell carcinoma. Int J Radiat Oncol Biol Phys 2005; 61(1): 129-36.

[4] Ay MR, Zaidi H. Assessment of errors caused by X-ray scatter and use of contrast medium when using CT-based attenuation correction in PET. Eur J Nucl Med Mol Imaging 2006; 33(11): 1301-13.

[5] Townsend DW, Ed. Categorical course in diagnostic radiology: clinical PET and PET/CT imaging - PET basics, physics and instrumentation for PET. RSNA refresher course. McCormick Place, Chicago, IL. 2007.

[6] Kinahan PE, Townsend DW, Beyer T, Sashin D. Attenuation correction for a combined 3D PET/CT scanner. Med Phys 1998; 25(10): 2046-53.

[7] Henkin RE. Nuclear medicine. 2nd ed. Philadelphia: Mosby Elsevier 2006.

[8] Bunyaviroch T, Turkington TG, Wong TZ, Wilson JW, Colsher JG, Coleman RE. Quantitative effects of contrast enhanced CT attenuation correction on PET SUV measurements. Mol Imaging Biol 2008; 10(2): 107-13.

[9] Mawlawi O, Erasmus JJ, Munden RF, et al. Quantifying the effect of IV contrast media on integrated PET/CT: clinical evaluation. AJR Am J Roentgenol 2006; 186(2): 308-19.

[10] Buther F, Stegger L, Dawood M, et al. Effective methods to correct contrast agent-induced errors in PET quantification in cardiac PET/CT. J Nucl Med 2007; 48(7): 1060-8.

[11] Sheskin DJ. Handbook of parametric and nonparametric statistical procedures. $4^{\text {th }}$ ed. Boca Raton: Chapman \& Hall 2007.

[12] Branstetter BF, Blodgett TM, Zimmer LA, et al. Head and neck malignancy: is PET/CT more accurate than PET or CT alone? Radiology 2005; 235(2): 580-6.

[13] Schoder H, Yeung HW. Positron emission imaging of head and neck cancer, including thyroid carcinoma. Semin Nucl Med 2004; 34(3): 180-97.

[14] Yoshida K, Suzuki A, Nagashima T, et al. Staging primary head and neck cancers with (18)F-FDG PET/CT: is intravenous contrast administration really necessary? Eur J Nucl Med Mol Imaging 2009; 36(9): 1417-24.

[15] Rodrigues RS, Bozza FA, Christian PE, et al. Comparison of whole-body PET/CT, dedicated high-resolution head and neck $\mathrm{PET} / \mathrm{CT}$, and contrast-enhanced $\mathrm{CT}$ in preoperative staging of clinically M0 squamous cell carcinoma of the head and neck. J Nucl Med 2009; 50(8): 1205-13.

[16] Heusner TA, Kuehl H, Veit-Haibach P, et al. Highly iodinated intravenous contrast material for PET/CT - a feasibility study. Rofo 2008; 180(8): 740-5. 
[17] Nehmeh SA, Erdi YE, Kalaigian H, et al. Correction for oral contrast artifacts in $\mathrm{CT}$ attenuation-corrected PET images obtained by combined PET/CT. J Nucl Med 2003; 44(12): 1940-4.

[18] Matsumoto K, Nakamoto Y, Sakamoto S, Murase K, Senda M. Feasibility of diagnostic contrast-enhanced CT for attenuation correction of whole body PET images. Nippon Hoshasen Gijutsu Gakkai Zasshi 2007; 63(7): 757-65.

[19] Yau YY, Chan WS, Tam YM, et al. Application of intravenous contrast in PET/CT: does it really introduce significant attenuation correction error? J Nucl Med 2005; 46(2): 283-91.

[20] Otero HJ, Yap JT, Patak MA, et al. Evaluation of low-density neutral oral contrast material in PET/CT for tumor imaging: results of a randomized clinical trial. AJR Am J Roentgenol 2009; 193(2): 326-32.

[21] Antoch G, Freudenberg LS, Stattaus J, et al. Whole-body positron emission tomography-CT: optimized CT using oral and IV contrast materials. AJR Am J Roentgenol 2002; 179(6): 1555-60.
[22] Berthelsen AK, Holm S, Loft A, Klausen TL, Andersen F, Hojgaard L. PET/CT with intravenous contrast can be used for PET attenuation correction in cancer patients. Eur J Nucl Med Mol Imaging 2005; 32(10): 1167-75.

[23] Rodriguez-Vigil B, Gomez-Leon N, Pinilla I, et al. PET/CT in lymphoma: prospective study of enhanced full-dose PET/CT versus unenhanced low-dose PET/CT. J Nucl Med 2006; 47(10): 1643-8.

[24] Juergens KU, Oei ML, Weckesser M, et al. Whole-body imaging of oncologic patients using 16-channel PET-CT. Evaluation of an i.v. contrast enhanced MDCT protocol. Nuklearmedizin 2008; 47(1): 30-6.

[25] Yamamoto Y, Wong TZ, Turkington TG, Hawk TC, Coleman RE. Head and neck cancer: dedicated FDG PET/CT protocol for detection--phantom and initial clinical studies. Radiology 2007; 244(1): 263-72.

(C) Hall et al.; Licensee Bentham Open.

This is an open access article licensed under the terms of the Creative Commons Attribution Non-Commercial License (http://creativecommons.org/licenses/by-nc/ $3.0 /$ ) which permits unrestricted, non-commercial use, distribution and reproduction in any medium, provided the work is properly cited. 